\section{A negative loop within the nuclear pore complex controls global chromatin organization}

\author{
Manuel Breuer and Hiroyuki Ohkura \\ The Wellcome Trust Centre for Cell Biology, School \\ of Biological Sciences, University of Edinburgh, Edinburgh \\ EH9 3JR, United Kingdom
}

The nuclear pore complex (NPC) tethers chromatin to create an environment for gene regulation, but little is known about how this activity is regulated to avoid excessive tethering of the genome. Here we propose a negative regulatory loop within the NPC controlling the chromatin attachment state, in which Nup155 and Nup93 recruit Nup62 to suppress chromatin tethering by Nup155. Depletion of Nup62 severely disrupts chromatin distribution in the nuclei of female germlines and somatic cells, which can be reversed by codepleting Nup155. Thus, this universal regulatory system within the NPC is crucial to control large-scale chromatin organization in the nucleus.

Supplemental material is available for this article.

Received April 30, 2015; revised version accepted August 12, 2015.

The nuclear pore complex (NPC) is the molecular hub for transport in and out of the nucleus. The NPC contains $\sim 30$ nucleoporins that are organized into distinct subcomplexes, including a core scaffold, peripheral nucleoporins, and central channel porins with characteristic phenylalanine-glycine (FG)-rich repeats (Alber et al. 2007). Embedded in the nuclear envelope, the NPC serves as a selective barrier to control nucleo-cytoplasmic, bidirectional transport (Floch et al. 2014).

Ever since a physical association of the NPC with the genome was postulated (Blobel 1985), increasing evidence has pointed to a role for the NPC in tethering chromatin to create an environment for gene regulation. Tethering specific genomic loci to the NPC appears to contribute to transcriptional activation (Casolari et al. 2004; Taddei et al. 2006; Light et al. 2010; Ikegami and Lieb 2013; see Pascual-Garcia and Capelson 2014). Also, the NPC has been further implicated in creating a repressive environment (Green et al. 2012; Van de Vosse et al. 2013) or retaining genes at the periphery after repression, possibly contributing to epigenetic transcriptional memory (Light et al. 2010). However, specific gene regulation modules aside, little is known about how the association of chromatin at a global scale is regulated to avoid excessive, unwanted attachment and how misregulation affects chromatin organization.

Here we propose that the NPC contains a regulatory circuit controlling the chromatin attachment state in the fe-

[Keywords: chromatin; nuclear pore; meiosis; Drosophila; karyosome] Corresponding author: h.ohkura@ed.ac.uk

Article is online at http://www.genesdev.org/cgi/doi/10.1101/gad.264341. 115. Freely available online through the Genes \&) Development Open Access option. male germline and somatic cells. Loss of an NPC subunit, Nup62 or Nup93, leads to excessive chromatin attachment to the nuclear envelope, which can be rescued by codepletion of a chromatin-binding NPC subunit, Nup155. This study highlights a major role of the NPC in global chromatin organization and suggests a universal regulatory system within the NPC.

\section{Results and Discussion}

NPC subunits Nup62 and Nup93 suppress excessive chromatin attachment to the nuclear envelope

Cytological study of the chromatin attachment state to the nuclear envelope is experimentally challenging, as chromatin usually occupies the entire nucleus. However, meiotic chromatin becomes fully detached from the nuclear envelope and compacted into a spherical structure, the karyosome, after recombination in Drosophila oocytes (Fig. 1A; King 1970). Chromatin detachment and karyosome formation are crucial to make a single spindle and allow subsequent chromosome segregation /Cullen et al. 2005) and are conserved features also seen in mammalian oocytes (Parfenov et al. 1988). By taking advantage of this unique nuclear organization in oocytes, we sought factors required for chromatin detachment from the nuclear envelope by individually knocking down various nuclear proteins in the female germline (the oocyte and nurse cells) by RNAi.

Strikingly, the depletion of either of two nuclear pore proteins, Nup62 or Nup93 (Supplemental Fig. S1A), led to disruption of the compact karyosome morphology (Fig. 1A,B), while the depletion of several other pore proteins did not (Supplemental Fig. S2). The chromatin shifted near the nuclear periphery, resulting in strong (Nup62) or partial (Nup93) overlap with a nuclear pore marker in the oocytes in comparison with the control RNAi (Fig. 1C), which was confirmed by superresolution microscopy (Supplemental Fig. S3). Nup93 is a linker scaffold protein known to be required for the recruitment of Nup62, one of the central channel proteins containing FG repeats (Grandi et al. 1993, 1995; Sachdev et al. 2012). We confirmed that the defect is not an off-target effect by rescue experiments using RNAi-resistant transgenes (Fig. 1B). In addition, similar karyosome defects were observed in female sterile Nup62 mutants that we generated (Fig. 1A; Supplemental Fig. S4A-C). In nurse cells (polytenized germline cells that support oocyte growth), chromatin also distributed irregularly and more toward the nuclear periphery after RNAi of these genes (Fig. 1D). This demonstrates a general role for both genes in global chromatin organization rather than being restricted to oocytes.

\section{Excessive chromatin attachment is independent of the meiotic checkpoint and is only associated with mild transport defects}

To identify the cause of the karyosome defect upon Nup62 or Nup93 RNAi, we first tested the structural integrity and

(C) 2015 Breuer and Ohkura This article, published in Genes e) Development, is available under a Creative Commons License (Attribution 4.0 International), as described at http://creativecommons.org/licenses/by/4.0/. 

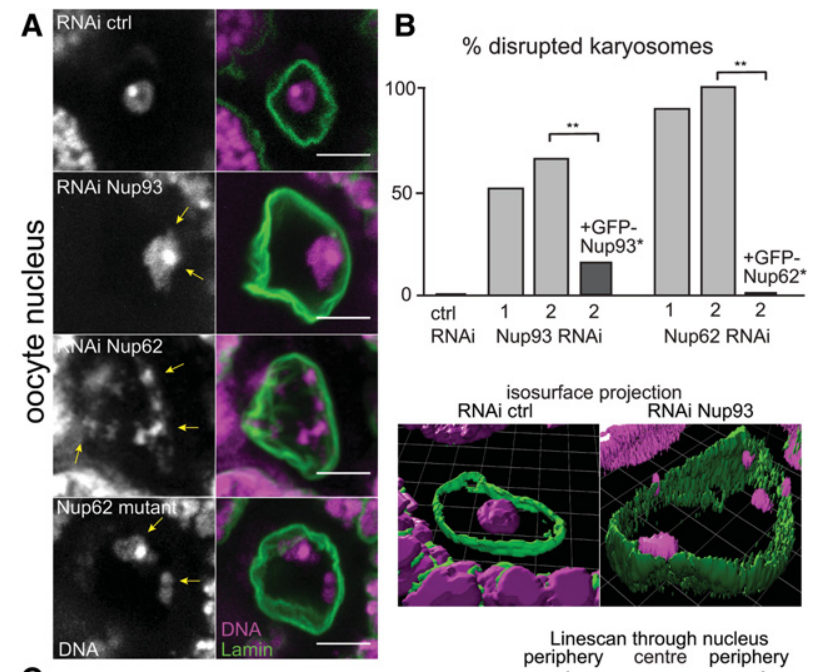

C

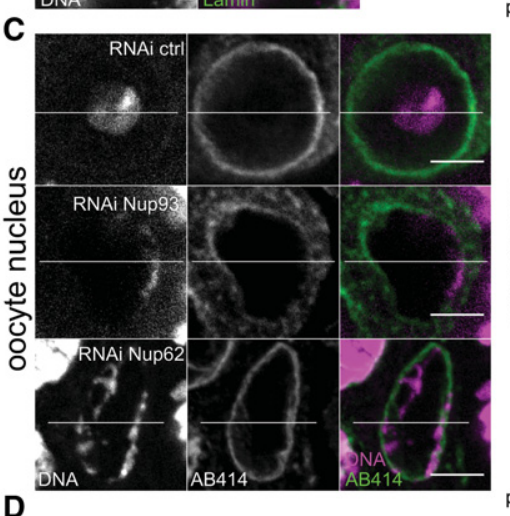

D
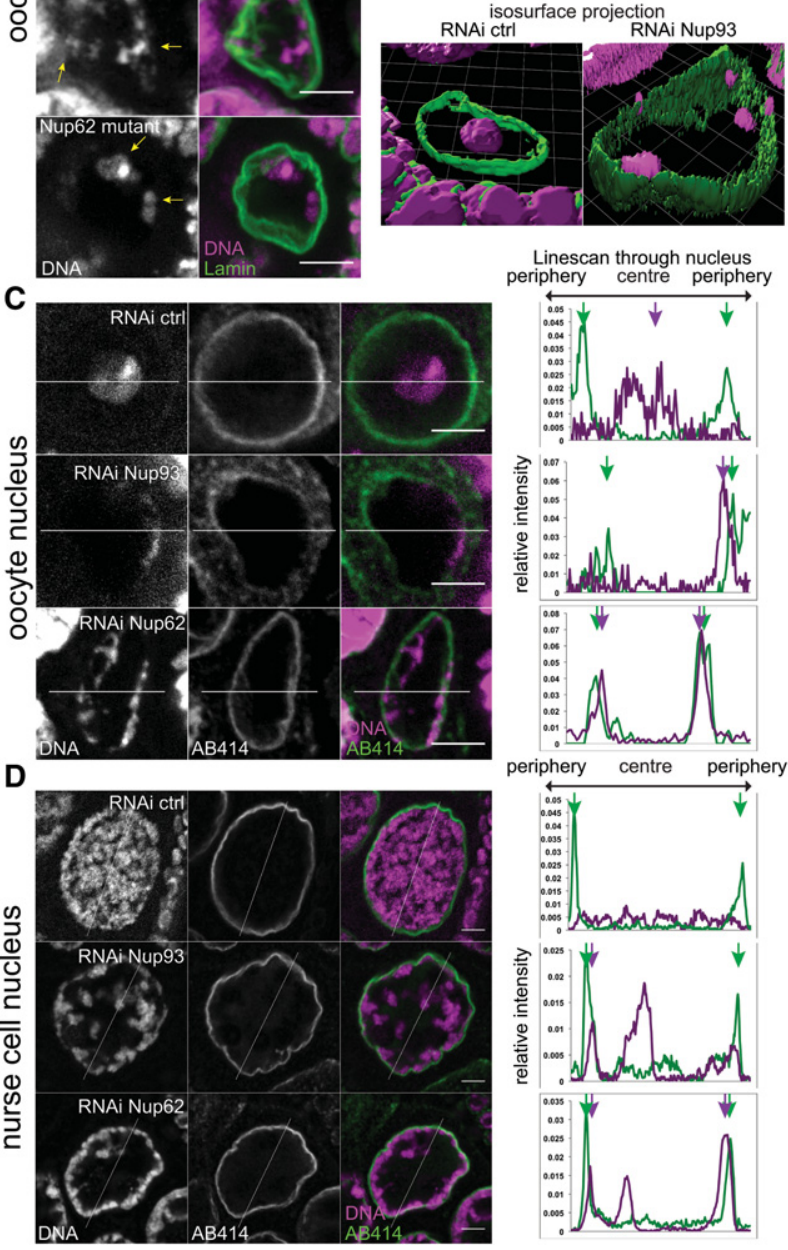

Linescan through nucleus
periphery centre periphery periphery centre peripher
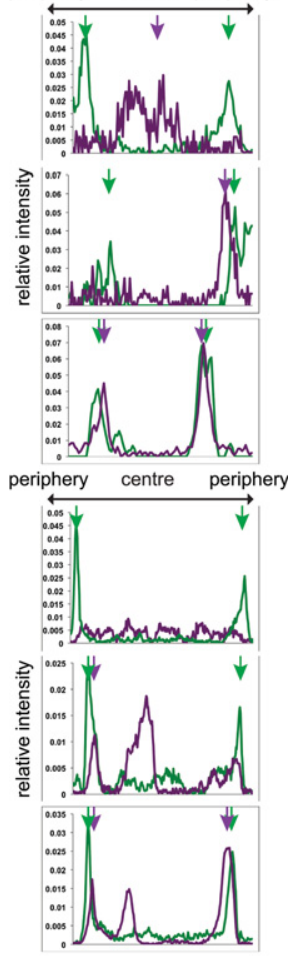

Figure 1. Excessive chromatin positioning at the nuclear periphery upon loss of Nup93 or Nup62 in oocytes and nurse cells. (A) The karyosome in the oocyte nucleus is proximal to the nuclear periphery (arrows) upon Nup93 or Nup62 RNAi or a Nup62 495 mutant. Bar, 5 um. (B) Frequencies of disrupted karyosomes in oocytes expressing a control shRNA or shRNA against Nup93 (column 1 or 2; nonoverlapping shRNA) or Nup62 (column 1 or 2) and in oocytes expressing shRNA2 and a GFP-tagged transgene resistant to RNAi $\left.\left({ }^{*}\right) .{ }^{* *}\right) P<0.0001 . n=35$ for control; $27 \leq n \leq 50$ for Nup93; $18 \leq n \leq 47$ for Nup62. (C,D) Maximum intensity projections of three midsections (total $1.5 \mu \mathrm{m}$ ) of oocyte $(C)$ or nurse cell $(D)$ nuclei expressing control shRNA or shRNA against Nup93 or Nup62 with relative signal intensities along a line. Arrows indicate peaks of DNA and nuclear pore signals (AB414).

transport function of NPC. RNAi of Nup62 or Nup93 did not disrupt the overall structural integrity of the NPC, as judged by the localization of FG-containing subunits and the core scaffold subunit Nup107 (Supplemental Fig. S5A-C). The active import function of the NPC showed small differences as assessed by fluorescence recovery after photobleaching (FRAP) of GFP fused with a nuclear localization signal (NLS) (Supplemental Fig. S5D). There was a significant increase in the nuclear size of early oocytes (Supplemental Fig. S5E), which may be caused by a reduced ability of the nuclear pore to act as a diffusion barrier.

Next, we examined a relationship with the meiotic recombination checkpoint, which is known to disrupt karyosome formation in the presence of unrepaired doublestrand breaks (DSBs) in oocytes (Ghabrial and Schüpbach 1999). Inactivation of the checkpoint did not suppress the karyosome defects of Nup62 or Nup93 RNAi (Supplemental Fig. S4D), demonstrating that the defect is independent of the meiotic recombination checkpoint in oocytes.

\section{NPC-interacting chromatin is enriched at the nuclear periphery upon Nup93 depletion}

Considering the above results, we hypothesized that chromatin is excessively anchored to the NPC in RNAi of Nup62 or Nup93. If this was the case, we predicted that chromatin specifically interacting with the NPC must be preferentially accumulated at the nuclear periphery rather than random chromatin (Fig. 2A). In order to test this, we used previously identified genomic loci bound to another nuclear pore component, Nup98, in Drosophila S2 culture cells (Kalverda et al. 2010). Nup98 has two distinct populations-one at nuclear pores and the other in the nucleoplasm - that bind distinct genomic loci in S2 cells (Kalverda et al. 2010). Nurse cells were subjected to fluorescence in situ hybridization (FISH) using individual probes corresponding to genomic loci known to be associated with Nup98 within the NPC or located in the nucleoplasm in S2 cells and were costained with a DNA dye (Fig. 2B). We measured a proportion of the total DNA signals in the nuclear periphery region, defined by a distance from the nuclear lamina of $<10 \%$ of the nuclear radius, which occupies $\sim 20 \%$ of the nuclear area. In control RNAi, $\sim 16 \%-17 \%$ of the total DNA (propidium iodide or DAPI signal) was located in the nuclear periphery region (Fig. 2C). For all genomic loci (three NPC-bound and four nucleoplasmic), $17 \%-25 \%$ of the signal foci were found in the nuclear periphery (Fig. 2C). This indicates that there is no preference for periphery locations of the total DNA or of these specific genomic regions in wild-type nurse cells. When Nup93 was knocked down, there was a small increase (from $16 \%-17 \%$ to $20 \%-$ $24 \%$ ) in the total DNA that occupies the nuclear periphery (Nup93 RNAi was used, since it gives a milder phenotype than Nup62 RNAi) (Fig. 2C). Strikingly, we observed a strong, consistent redistribution of all NPC-bound genomic loci to the periphery (from $17 \%-25 \%$ to $>40 \%$ ), whereas the nucleoplasmic loci showed smaller variable changes (Fig. 2C). The increases for NPC-bound loci were significantly higher than the increases for both total DNA and the nucleoplasmic loci, supporting our hypothesis that depletion of Nup62 or Nup93 results in an excessive attachment of specific chromatin regions to the NPC.

Nup155 is required for chromatin attachment to the nuclear envelope in the absence of Nup62 or Nup93

Our results suggest that Nup62 and Nup93 suppress the interaction between chromatin and another NPC subunit. If this is the case, codepletion of this hypothetical NPC 
A

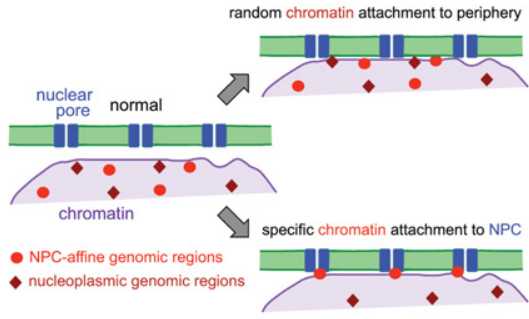

B

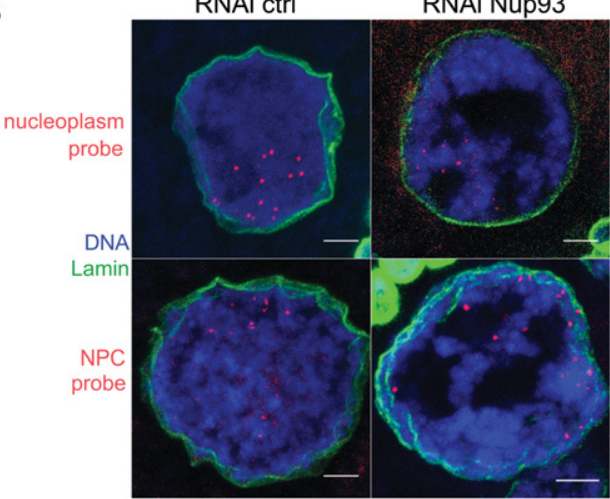

C Signals at nuclear periphery

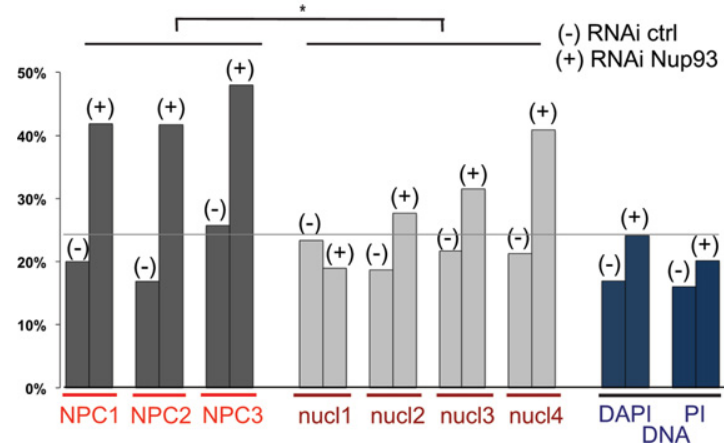

Figure 2. NPC-interacting chromatin is more frequently located at the nuclear periphery without Nup93. (A) Nuclear pore-interacting genomic regions found in $\mathrm{S} 2$ cells migrate more closely to the nuclear periphery than nucleoplasmic genomic regions upon Nup93 depletion if the pores excessively tether chromatin. $(B)$ FISH signals for known NPC-bound regions were more frequently located at the nuclear periphery than those for nucleoplasmic regions when Nup93 is depleted as compared with a control. Bars, $5 \mu \mathrm{m}$. $(C)$ Percentages of FISH and DAPI/propidium iodide (PI) signals in the nuclear periphery (the distance from the nuclear lamina is $<10 \%$ of the nuclear radius) in nurse cells subjected to control and Nup93 RNAi. (-) Control RNAi $_{i}(+)$ Nup93 RNAi. $79 \leq n \leq 203$. Changes from control RNAi to Nup93 RNAi were significantly greater for the three NPC probes than the four nucleoplasmic probes. $\left({ }^{*}\right) P=0.0179$.

subunit that mediates chromatin attachment to the nuclear pore should restore detachment of chromatin in Nup62or Nup93-depleted oocytes. Several NPC subunits have previously been shown to have chromatin-binding activity, including Nup155, Nup50, and ELYS/Mel-28 (Gillespie et al. 2007; Kalverda et al. 2010; Busayavalasa et al. 2012). We generated flies expressing two shRNAs: one for Nup62 and the other for each of the aforementioned chromatin-binding NPC subunits, the non-chromatin-bound Nup160, or a control (Fig. 3A; Supplemental Fig. S1B). Codepletion of Nup155 specifically restored normal karyosome morphology and detachment from the nuclear periphery in Nup62-depleted oocytes (Fig. 3A,B). Furthermore, in nurse cells, simultaneous RNAi of Nup155 also restored normal chromatin distribution caused by Nup62 RNAi (Fig. 3C). Crucially, codepletion of Nup155 did not rescue the larger nuclear size in Nup62-depleted oocytes. This demonstrates that Nup62's function on chromatin organization is independent of its function on nuclear size maintenance (Supplemental Fig. S6A), which may reflect its function as a diffusion barrier.

We also found that single depletion of Nup155 led to a large reduction of Nup62 (one of the FG-containing subunits) from the nuclear envelope and its accumulation in the cytoplasm. However, it did not significantly reduce the total amount of the FG-containing subunits at the nuclear envelope in both Nup155 and Nup62/Nup155 double RNAi (Supplemental Fig. S6B-D). This demonstrates that Nup155 is required for Nup62 recruitment, and the apparent rescue of the Nup62 depletion defect by Nup 155 codepletion is not due to a loss of integrity or a reduced number of nuclear pores. Taken together, the results suggest a negative regulatory loop in which Nup155 recruits Nup62 to the nuclear pores, and, in turn, Nup62 suppresses chromatin anchoring by Nup155.

\section{Somatic cells harbor a common regulatory circuit that controls chromatin distribution}

We uncovered a potential negative regulatory circuit within the NPC that controls the chromatin attachment state to the nuclear pores in the oocytes and nurse cells. Therefore, we sought to test whether a common

A

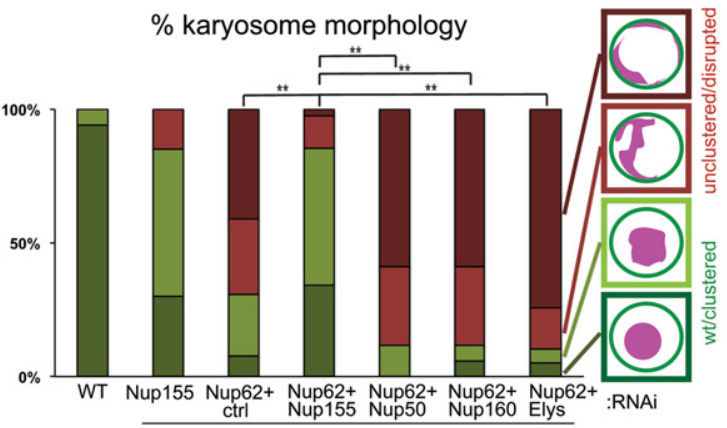

B

oocyte nucleus

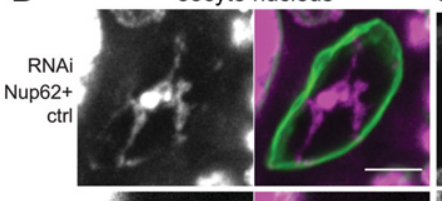

C nurse cell nucleus

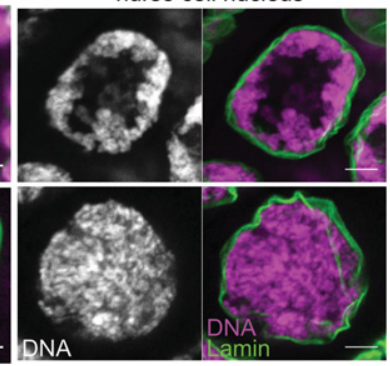

Figure 3. Codepletion of Nup155 rescued excessive chromatin attachment to the nuclear periphery caused by Nup62 depletion. $(A)$ Karyosome morphology was classified into four categories, from normal to severely defective (diagrams at the right). For double RNAi of control + Nup62, Nup155 + Nup62, and ELYS + Nup62, $39 \leq n \leq 41$; for double RNAi of Nup50 + Nup62 and Nup160+Nup62, $n=17$. The combined proportion of the two severe categories in Nup62+ Nup155 double RNAi was significantly lower than in others. (**) $P$ $=0.0001$. $(B, C)$ Restored chromatin morphology in the oocyte $(B)$ and nurse cell $(C)$ nuclei upon Nup62 and Nup155 double RNAi in comparison with Nup62 and control RNAi. Bar, $5 \mu \mathrm{m}$. 
regulatory system also controls chromatin organization in somatic cells. Using the Drosophila S2 cell line, we depleted Nup62 (Supplemental Fig. S6E) or Nup155 individually and simultaneously by RNAi. Control RNAi cells showed a relatively even distribution of chromatin within the nucleus except for a dense region that corresponds to heterochromatin (Kellum et al. 1995). In contrast, Nup62 RNAi resulted in an uneven distribution of chromatin within the nucleus (Fig. 4A). To quantify this, we measured the area that chromatin occupies relative to the nuclear area. The cells depleted of Nup62 showed a significant decrease in chromatin occupancy compared with a control RNAi (Fig. 4A,B). Strikingly, double depletion of Nup62 and Nup155 showed a chromatin occupancy similar to the control (Fig. 4A,B). This rescue was reversed by RNAi-resistant full-length Nup155 but not by resistant Nup155 lacking the chromatin-binding region (Fig. 4A,B; Busayavalasa et al. 2012). No significant change in chromatin occupancy was observed upon Nup155 depletion alone (Fig. 4A,B). This demonstrated the presence of a common negative loop within the NPC that controls the global chromatin distribution between female germline cells and somatic cells.

\section{A negative loop within the NPC controls global chromatin organization}

Recent reports described the role of the NPC to tether chromatin and thus create an environment for gene regu-

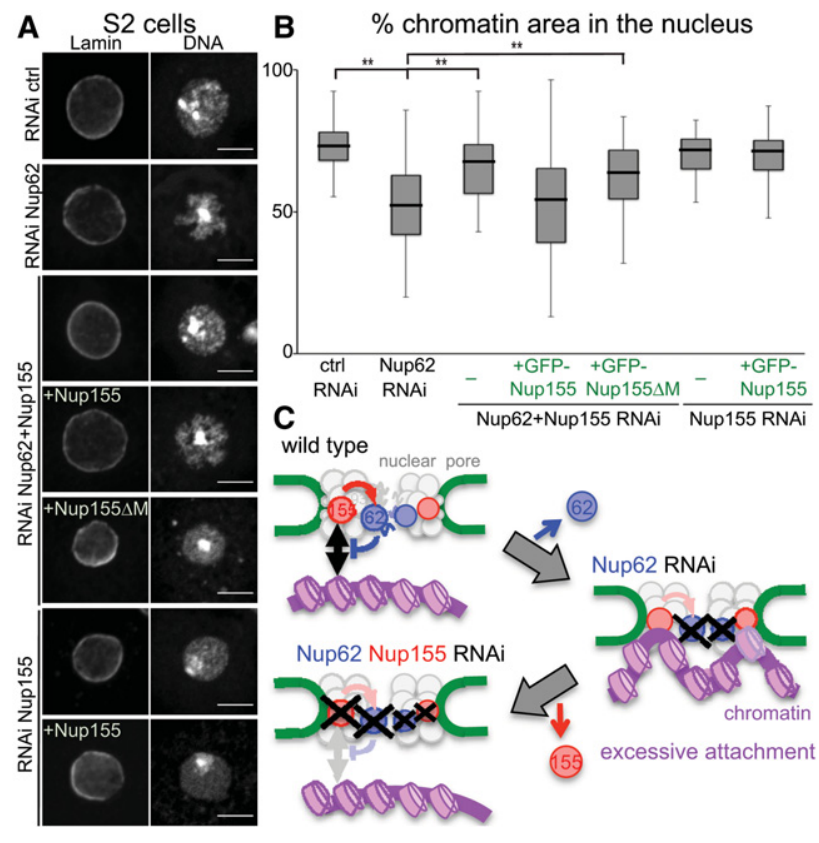

Figure 4. Loss of Nup62 disturbs chromatin distribution in somatic cells, which is rescued by codepletion of Nup155. (A) RNAi-treated (control, Nup62, Nup155, or Nup62 + Nup155) S2 cells were immunostained for Lamin and DNA. Some batches transiently expressed GFP-tagged RNAi-resistant full-length Nup155 (Nup155) and Nup155 lacking the chromatin-binding region (Nup155 $\Delta \mathrm{M})$. Bar, 5 $\mu \mathrm{m}$. (B) The proportion of the area occupied by chromatin compared with the entire nuclear area (Lamin). The box represents the middle two quartiles, and the whiskers represent the top and bottom quartiles. $23 \leq n \leq 48 .\left(^{* *}\right) P<0.01$, a significant difference from Nup62 RNAi. (C) Model for the chromatin attachment state controlled by an internal regulatory circuit in the NPC. lation (Taddei et al. 2006; Brown et al. 2008; Light et al. 2010; Kalverda et al. 2010; Ikegami and Lieb 2013; Van de Vosse et al. 2013; Pascual-Garcia and Capelson 2014). While recruitment mechanisms for specific genes have been described (Rodríguez-Navarro et al. 2004; Schmid et al. 2006; Light et al. 2010; Van de Vosse et al. 2013), very little is known about whether or how this tethering is regulated. This study makes two major conceptual advances in our understanding of global chromatin organization, especially the critical role and regulation of the NPCmediated tethering. First, it highlights a far greater role of the NPC in large-scale chromatin organization than previously anticipated. Second, it points to a universal regulatory circuit inside the NPC that controls the attachment state of chromatin to the nuclear pore. This consists of a negative regulatory loop in which chromatin-binding Nup155 recruits the central channel protein Nup62, which in turn suppresses chromatin binding (Fig. 4C). As nuclear pore components associate with the genome to positively or negatively influence gene expression (Pascual-Garcia and Capelson 2014; Ptak et al. 2014), this regulatory loop might be part of a wider network for the NPC to control gene expression, depending on the cellular and developmental context. Although a genuine and direct regulatory role of this loop has yet to be demonstrated, its intrinsic capacity supplies the NPC with a key mechanism to globally or locally organize the metazoan genome. On the other hand, any change or imbalance in this regulatory network might have dramatic effects for the nuclear architecture and, concomitantly, the expression profile of the cell. This may have a significant medical implication, as nuclear pore components not only are known to deteriorate with age but are also affected in several tissue-specific human diseases (Cronshaw and Matunis 2004; Capelson and Hetzer 2009; D'Angelo et al. 2009).

\section{Materials and methods}

Drosophila genetics

Standard fly techniques were followed (Ashburner et al. 2005). Flies were cultured at $25^{\circ} \mathrm{C}$ in standard cornmeal medium. For RNAi in ovaries, P\{Gal4::VP16-nos.UTR\}MVD1 or P\{MatTubulin67C-Gal4\}V37 flies were crossed with the following RNAi TRiP (Transgenic RNAi Project) lines (Harvard Medical School): Nup93 (HMS00850 and HMS00898), Nup62 (GLV21060 and GL01533), Nup155 (DmNup154; HMS01189), Nup50 (HMS01054), Nup160 (HMS00385), and ctrl-attP2 (P\{CaryP\}attP2); ctrl- $w^{+}$ (GL00094). GFP-Nup107 (P\{GFP-Nup107.K/9.1) (Katsani et al. 2008) and GFP-NLS (P\{Ubi-GFP.nls\}ID-2; P\{Ubi-GFP.nls\}ID-3I) were also used.

Nup62 mutants were generated by remobilization of P element (GSV1GS2186). The transposase gene $\Delta 2-3$ was crossed in, and chromosomes that had lost the $w^{+}$gene were tested over a deficiency $[D f(2 R) B S C 550$ or $D f(2 R)$ Exel6063] for viability and female fertility. Viable chromosomes were tested over the deficiency for the presence of the initiation codon of Nup62 by PCR. Plasmids containing UASp-GFP-Nup93* and UASp-GFPNup62* (the asterisk indicates shRNA-resistant) or Walium22-vectors (Harvard Medical School) containing shRNA against Mel-28/ELYS were injected into $w$ embryos carrying an attP40 or VK33 landing site by Genetic Research, Inc., for transgenesis.

The meiotic recombination checkpoint was suppressed by a heterozygous mutation, $m n k^{\mathrm{p} 6}$ (DmChk2) (Klattenhoff et al. 2007), or by feeding adults with medium containing spectral ice blue dye (Sugarflair Colours Ltd.) and $1 \mathrm{mg} / \mathrm{mL}$ caffeine (Sigma). spn ${ }^{A 1}$ (Tearle and Nüsslein-Volhard 1987) was used as control.

\section{Drosophila S2 cells}

Culture of S2 cells and RNAi were carried out as previously described (Dzhindzhev et al. 2005). For RNAi, S2 cells were incubated for 5-7 d 
with dsRNA against Nup62, the control $\beta$-lactamase gene, or the $5^{\prime}$ untranslated region (UTR) of Nup155. Some cells were transfected $2 \mathrm{~d}$ before fixation with a plasmid containing the metallothionein promoter followed by the Nup155 or Nup155 $\Delta$ M (540-958 amino acids were replaced by SASA) coding sequence.

\section{Molecular techniques}

Standard techniques were used throughout (Sambrook et al. 1989). Nup93and Nup62-coding regions were introduced into the Gateway (Invitrogen) entry vector pDONR221 and then into the destination vector $\phi \mathrm{PGW}$ (pPGW carrying the $\phi C 31$ attB site; UASp-GFP) or pMAL-Gateway (MBP fusion). To create the expression constructs resistant to the shRNAs, silent mutations were introduced (Nup93, GACAACTTG $>$ GATAATTTA; Nup62, ATCGTCGAG > ATAGTTGAA) using QuickChange XLII sitedirected mutagenesis kit (Agilent). To generate a Walium22-based plasmid that expresses shRNA targeting ELYS/Mel-28, the TRiP protocol (http://www.flyrnai.org/supplement/2ndGenProtocol.pdf) was followed using the oligonucleotides CTAGCAGTGCACTGTGCTGTTGGTTGA TCTAGTTATATTCAAGCATAGATCAACCAACAGCACAGTGCGCG and AATTCGCGCACTGTGCTGTTGGTTGATCTATGCTTGAATATA ACTAGATCAACCAACAGCACAGTGCACTG, respectively.

RT-qPCR was carried out as previously described (Nikalayevich and Ohkura 2015) except three ovary pairs from adult females (matured for $3-5 \mathrm{~d}$ at $25^{\circ} \mathrm{C}$ ) were used. Actin5C was used as a control for normalization.

\section{Cytological techniques}

Ovaries and S2 cells were immunostained and analyzed according to Lancaster et al. (2007) and Dzhindzhev et al. (2005). Primary antibodies were used as follows: mAB414 (mouse, 1:200-500 for immunofluorescence; Covance/Biolegend) (Aris and Blobel 1989), HP1 and Lamin (mouse C1A9 1:250 for Western blot, and mouse ADL67.10 1:200-250 for immunofluorescence; Developmental Studies Hybridoma Bank), and antiNup62 (rat 1:200-500 for immunofluorescence and 1:250 for Western blot) (this study). Nup62 antibodies were raised against MBP-Nup62 purified from Escherichia coli, and the final bleed was used. Secondary antibodies $(1: 15,000)$ were purchased from Jackson Immunologicals, Molecular Probes, or Odyssey (Li-Cor) and used as detected by an Odyssey scanner (Li-Cor) for Western blot. For live imaging, oocytes were dissected from matured adult females in halocarbon oil (700) and observed using a spinning disc confocal microscope (UltraView, Perkin Elmer). Typically, a series of $Z$-sections (separated by $0.5 \mu \mathrm{m}$ ) was taken for ovarioles. For live DNA visualization, ovaries were washed in medium containing 5 $\mu$ M DRAQ5 (Biostatus Ltd.) and mounted in oil. Single Z-planes or maximum intensity $Z$-projections of selected planes are shown in the figures after contrast was adjusted uniformly across the field using ImageJ (National Institutes of Health). For structured illumination microscopy (SIM), a Nikon N-SIM (Nikon) with a water immersion objective $(60 \times, 1.2$ NA; Nikon) and a xIon897 EMCCD camera (the gain at 300; Andor) was used. Images were reconstructed using the Nikon N-SIM software, and the measured resolution in the final image was $160-170 \mathrm{~nm}$. For the reconstruction, the Wiener filter and apodization filter parameters were kept constant for all samples (Gustafsson et al. 2008).

\section{FRAP experiments}

Ovaries expressing shRNA and GFP-NLS were observed in halocarbon oil. A region of interest (ROI) corresponding to the nucleus was drawn on the mid $Z$-plane of the oocyte nucleus (stages 4-6). Images were acquired using laser power $5 \%$, gain 650 , and pinhole with 6.2 airy units every $8 \mathrm{sec}$ for 40 time points. After five time points, the ROI was photobleached by 488-nm laser (100\% power) with 25 iterations. The average intensity for each oocyte nucleus was corrected using the nurse cell nucleus as a nonphotobleached control. Averages for all nuclei in sample groups were entered into the equation for a fitted curve of expected recovery, and $t 1 / 2$, as time needed to reach recovery equal to one-half maximum recovery, was determined as described (Colombié et al. 2013).

\section{FISH}

The following $\mathrm{CH} 322$-based BACs containing the genomic regions that bind NPC-tethered Nup98 and nucleoplasmic Nup98 in S2 cells
(NPC1-3 and nucleoplasm1-4) (Kalverda et al. 2010) were used for FISH: 53P20, 169H03, and 176O04 + 12I13 were used for NPC1-3, and 120G02 + 175O14, 70N20, 51D05, and 92H12 were used for nucleoplasmic1-4 (BACPAC Resources Center). BACs were digested with AluI, HaeIII, MseI, MspI, RsaI, and Sau3AI in 4BC buffer + BSA + DTT. DNA was precipitated and resuspended in TE. Ten micrograms of DNA was denatured for 2 min at $95^{\circ} \mathrm{C}$ and put on ice before labeling with ChromaTide Alexa-564 dUTP and unlabeled dTTP (1:8 ratio) using $60 \mathrm{U}$ of terminal deoxynuleotidyl transferase (TdT) in TdT buffer for $1 \mathrm{~h}$ at $37^{\circ} \mathrm{C}$. Glycogen was added, and probes were subjected to a Sephadex G-25 column before being precipitated, washed, and resuspended in TE. Oocytes were fixed in prewarmed $3.7 \%$ formaldehyde for $4 \mathrm{~min}$ and washed in $2 \times$ SSCT. Oocytes were gradually put into $2 \times \mathrm{SSCT} / 50 \%$ formamide and incubated for $2-4 \mathrm{~h}$ at $37^{\circ} \mathrm{C}$ before incubating in $1.1 \times$ hybridization buffer with 50-600 ng of the FISH probe (final $40 \mu \mathrm{L}$ ) at $37^{\circ} \mathrm{C}$. Samples were subsequently washed out of $2 \times$ SSCT/formamide and, after final washes, in $2 \times$ SSCT, followed by immunostaining protocol.

\section{Image analysis}

To generate line-scan intensity profiles, images were background-subtract$\mathrm{ed}$, and several $Z$-stacks were combined to a maximum projection. A line was drawn across the nucleus, and its plot profile was measured by ImageJ and normalized in relation to total values for AB414 and DAPI along the line.

To quantify the occupancy of DNA distribution in nurse cell nuclei in two dimensions (FISH experiments), we used a macro written for Image Pro Plus (D. Kelly, Wellcome Trust Centre for Cell Biology) to measure the total nuclear area on the DAPI images (manually intensity-thresholded and background-subtracted) and then divided the DAPI-defined nucleus into five shells of equal area. The percentage of DAPI signal was estimated in the outermost shell, which occupied $20 \%$ in area or $\sim 10 \%$ of the nuclear radius. For the FISH probes, we took measurements for the three most central Z-stacks of every nucleus. The average of three lines from the center to the Lamin signal was used as the radius of each nucleus. Positions of FISH signals were measured as the shortest distance to the nearest point of the Lamin staining, and the proportion of the signals within $10 \%$ of the nuclear radius from the periphery was estimated.

To measure the area occupied by chromatin in S2 cell nuclei, we used DAPI and Lamin immunostaining images taken using the same setting and subtracted the background (Image). The DAPI images were manually thresholded to the highest background value in the cytoplasm, and areas were measured with a particle size setting of 9 to infinity. Total area values for DAPI were calculated relative to total nuclear area defined by Lamin.

The diameters of the oocyte nuclei were measured by choosing the mid$Z$-plane of the nucleus and averaging two measurements of the nuclear diameter in a $90^{\circ}$ angle to each other. The signal intensity of AB414 and Nup62 at the nuclear envelope was determined on a maximum projection image of three $Z$-stacks using the formula $\left(I_{\mathrm{NE}}-I_{\mathrm{cyt}}\right) / I_{\mathrm{cyt}}$, where $I_{\mathrm{cyt}}$ and $I_{\mathrm{NE}}$ were defined as the averages of each maximum intensity in three boxes with a defined size in the cytoplasm (background) and on the nuclear envelope (signal), respectively.

Fisher's exact test, Wilcoxon test, and $t$-test were used for categorical, nonparametric, and parametric data, respectively.

\section{Acknowledgments}

We thank the Bloomington Drosophila Stock Center and Resource Center (National Institutes of Health P40OD018537 and 2P40OD010949-10A1), the Kyoto Drosophila Genetic Resource Center, and the Transgenic RNAi Project (TRiP) at Harvard Medical School (National Institutes of Health/National Institute of General Medical Sciences R01-GM084947) for fly stocks and reagents. We especially thank D. Kelly (Centre Optical Instrumentation Laboratory [COIL]), S. Webb (COIL), S. Beard, R. Beaven, F. Cullen, P. Romé, and A. Wheeler (Institute of Genetics and Molecular Medicine, Edinburgh Super-resolution Imaging Consortium) for technical assistance. We also thank P. Heun, E. Schirmer, and all members of the Ohkura laboratory for critical reading of the manuscript. This work is supported by The Wellcome Trust (081849, 092076, and 098030). 


\section{References}

Alber F, Dokudovskaya S, Veenhoff LM, Zhang W, Kipper J, Devos D, Suprapto A, Karni-Schmidt O, Williams R, Chait BT, et al. 2007. The molecular architecture of the nuclear pore complex. Nature 450: 695-701.

Aris JP, Blobel G. 1989. Yeast nuclear envelope proteins cross-react with an antibody against mammalian pore complex proteins. J Cell Biol 108: 2059-2067.

Ashburner M, Golic KG, Hawley RS. 2005. Drosophila: a laboratory handbook. Cold Spring Harbor Laboratory Press, Cold Spring Harbor, NY.

Blobel G. 1985. Gene gating: a hypothesis. Proc Natl Acad Sci 82: 8527-8529.

Brown CR, Kennedy CJ, Delmar VA, Forbes DJ, Silver PA. 2008. Global histone acetylation induces functional genomic reorganization at mammalian nuclear pore complexes. Genes Dev 22: 627-639.

Busayavalasa K, Chen X, Farrants AK, Wagner N, Sabri N. 2012. The Nup155-mediated organisation of inner nuclear membrane proteins is independent of Nup155 anchoring to the metazoan nuclear pore complex. J Cell Sci 125: 4214-4218.

Capelson M, Hetzer MW. 2009. The role of nuclear pores in gene regulation, development and disease. EMBO Rep 10: 697-705.

Casolari JM, Brown CR, Komili S, West J, Hieronymus H, Silver PA. 2004. Genome-wide localization of the nuclear transport machinery couples transcriptional status and nuclear organization. Cell 117: 427-439.

Colombié N, Głuszek AA, Meireles AM, Ohkura H. 2013. Meiosis-specific stable binding of Augmin to acentrosomal spindle poles promotes biased microtubule assembly in oocytes. PLoS Genet 9: e1003562.

Cronshaw JM, Matunis MJ. 2004. The nuclear pore complex: disease associations and functional correlations. Trends Endocrinol Metab 15: 34-39.

Cullen CF, Brittle AL, Ito T, Ohkura H. 2005. The conserved kinase NHK1 is essential for mitotic progression and unifying acentrosomal meiotic spindles in Drosophila melanogaster. J Cell Biol 171: 593-602.

D'Angelo MA, Raices M, Panowski SH, Hetzer MW. 2009. Age-dependent deterioration of nuclear pore complexes causes a loss of nuclear integrity in postmitotic cells. Cell 136: 284-295.

Dzhindzhev NS, Rogers SL, Vale RD, Ohkura H. 2005. Distinct mechanisms govern the localisation of Drosophila CLIP-190 to unattached kinetochores and microtubule plus-ends. J Cell Sci 118: 3781-3790.

Floch AG, Palancade B, Doye V. 2014. Fifty years of nuclear pores and nucleocytoplasmic transport studies: multiple tools revealing complex rules. Methods Cell Biol 122: 1-40.

Ghabrial A, Schüpbach T. 1999. Activation of a meiotic checkpoint regulates translation of Gurken during Drosophila oogenesis. Nat Cell Biol 1: 354-357.

Gillespie PJ, Khoudoli GA, Stewart G, Swedlow JR, Blow JJ. 2007. ELYS/ MEL-28 chromatin association coordinates nuclear pore complex assembly and replication licensing. Curr Biol 17: 1657-1662.

Grandi P, Doye V, Hurt EC. 1993. Purification of NSP1 reveals complex formation with 'GLFG' nucleoporins and a novel nuclear pore protein NIC96. EMBO I 12: 3061-3071.

Grandi P, Schlaich N, Tekotte H, Hurt EC. 1995. Functional interaction of Nic96p with a core nucleoporin complex consisting of Nsplp, Nup49p and a novel protein Nup57p. EMBO J 14: 76-87.

Green EM, Jiang Y, Joyner R, Weis K. 2012. A negative feedback loop at the nuclear periphery regulates GAL gene expression. Mol Biol Cell 23: 1367-1375.

Gustafsson MG, Shao L, Carlton PM, Wang CJ, Golubovskaya IN, Cande WZ, Agard DA, Sedat JW. 2008. Three-dimensional resolution dou- bling in wide-field fluorescence microscopy by structured illumination. Biophys J 94: 4957-4970.

Ikegami K, Lieb JD. 2013. Integral nuclear pore proteins bind to Pol III-transcribed genes and are required for Pol III transcript processing in C. elegans. Mol Cell 51: 840-849.

Kalverda B, Pickersgill H, Shloma VV, Fornerod M. 2010. Nucleoporins directly stimulate expression of developmental and cell-cycle genes inside the nucleoplasm. Cell 140: 360-371.

Katsani KR, Karess RE, Dostatni N, Doye V. 2008. In vivo dynamics of Drosophila nuclear envelope components. Mol Biol Cell 19: 3652 3666.

Kellum R, Raff JW, Alberts BM. 1995. Heterochromatin protein 1 distribution during development and during the cell cycle in Drosophila embryos. J Cell Sci 108: 1407-1418.

King RC. 1970. Ovarian development in Drosophila melanogaster. Academic Press, New York.

Klattenhoff C, Bratu DP, McGinnis-Schultz N, Koppetsch BS, Cook HA, Theurkauf WE. 2007. Drosophila rasiRNA pathway mutations disrupt embryonic axis specification through activation of an ATR/Chk2 DNA damage response. Dev Cell 12: 45-55.

Lancaster OM, Cullen CF, Ohkura H. 2007. NHK-1 phosphorylates BAF to allow karyosome formation in the Drosophila oocyte nucleus. J Cell Biol 179: 817-824.

Light WH, Brickner DG, Brand VR, Brickner JH. 2010. Interaction of a DNA zip code with the nuclear pore complex promotes H2A.Z incorporation and INO1 transcriptional memory. Mol Cell 40: 112-125.

Nikalayevich N, Ohkura H. 2015. The NuRD nucleosome remodelling complex and NHK-1 kinase are required for chromosome condensation in oocytes. J Cell Sci 128: 566-575.

Parfenov V, Potchukalina G, Dudina L, Kostyuchek D, Gruzova M. 1988. Human antral follicles: oocyte nucleus and the karyosphere formation (electron microscopic and autoradiographic data). Gamete Res 22: 219-231.

Pascual-Garcia P, Capelson M. 2014. Nuclear pores as versatile platforms for gene regulation. Curr Opin Genet Dev 25: 110-117.

Ptak C, Aitchison JD, Wozniak RW. 2014. The multifunctional nuclear pore complex: a platform for controlling gene expression. Curr Opin Cell Biol 28: 46-53.

Rodríguez-Navarro S, Fischer T, Luo MJ, Antúnez O, Brettschneider S, Lechner J, Pérez-Ortín JE, Reed R, Hurt E. 2004. Sus1, a functional component of the SAGA histone acetylase complex and the nuclear pore-associated mRNA export machinery. Cell 116: 75-86.

Sachdev R, Sieverding C, Flötenmeyer M, Antonin W. 2012. The C-terminal domain of Nup93 is essential for assembly of the structural backbone of nuclear pore complexes. Mol Biol Cell 23: 740-749.

Sambrook J, Fritsch E, Maniatis T. 1989. Molecular cloning: a laboratory manual. Cold Spring Harbor Laboratory Press, Cold Spring Harbor, NY.

Schmid M, Arib G, Laemmli C, Nishikawa J, Durussel T, Laemmli UK. 2006. Nup-PI: the nucleopore-promoter interaction of genes in yeast. Mol Cell 21: 379-391.

Taddei A, Van Houwe G, Hediger F, Kalck V, Cubizolles F, Schober H, Gasser SM. 2006. Nuclear pore association confers optimal expression levels for an inducible yeast gene. Nature 441: 774-778.

Tearle R, Nüsslein-Volhard C. 1987. Tübingen mutants stocklist. Dros Inform Serv 66: 209-226.

Van de Vosse DW, Wan Y, Lapetina DL, Chen WM, Chiang JH, Aitchison JD, Wozniak RW. 2013. A role for the nucleoporin Nup170p in chromatin structure and gene silencing. Cell 152: 969-983. 


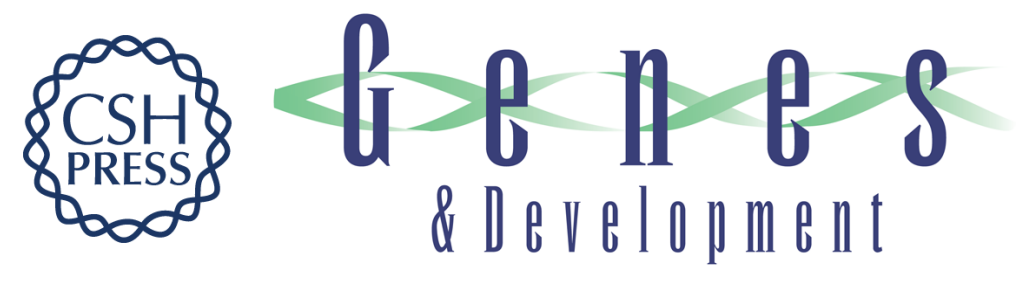

\section{A negative loop within the nuclear pore complex controls global chromatin organization}

Manuel Breuer and Hiroyuki Ohkura

Genes Dev. 2015, 29:

Access the most recent version at doi:10.1101/gad.264341.115

\section{Supplemental http://genesdev.cshlp.org/content/suppl/2015/09/04/29.17.1789.DC1 \\ Material}

References This article cites 36 articles, 13 of which can be accessed free at:

http://genesdev.cshlp.org/content/29/17/1789.full.html\#ref-list-1

Creative This article, published in Genes \& Development, is available under a Creative Commons

Commons License (Attribution 4.0 International), as described at

License http://creativecommons.org/licenses/by/4.0/.

Email Alerting Receive free email alerts when new articles cite this article - sign up in the box at the top Service right corner of the article or click here.

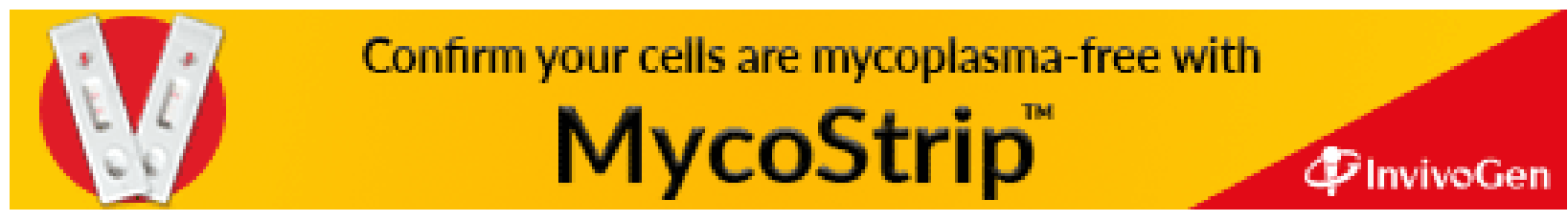

